\title{
Analysis of ZNF350/ZBRK1 promoter variants and breast cancer susceptibility in non-BRCA1/2 French Canadian breast cancer families
}

\author{
Karine V Plourde, Yvan Labrie, Sylvie Desjardins, Pascal Belleau, Geneviève Ouellette, \\ Francine Durocher and INHERIT BRCAs
}

\begin{abstract}
ZNF350/ZBRK1 is a transcription factor, which associates with BRCA1 to co-repress GADD45A to regulate DNA damage repair, and the expression of ZNF350 is altered in different human carcinomas. In a previous study, we identified ZNF350 genomic variants potentially involved in breast cancer susceptibility in high-risk non-BRCA1/2 breast cancer individuals, which pointed toward a potential association for variants in the $5^{\prime}$-UTR and promoter regions. Therefore, direct sequencing was undertaken and identified 12 promoter variants, whereas haplotype analyses put in evidence four common haplotypes with a frequency $>2 \%$. However, based on their frequency observed in breast cancer and unrelated healthy individuals, these are not statistically associated with breast cancer risk. Luciferase promoter assays in two breast cancer cell lines identified two haplotypes ( $\mathrm{H} 11$ and $\mathrm{H} 12$ ) stimulating significantly the expression of ZNF350 transcript compared with the common haplotype H8. The high expression of the $\mathrm{H} 11$ allele was associated with the variant c.-874A. Using Matlnspector and Transcription Element Search softwares, in silico analyses predicted that the variant c.-874A created a binding site for the factors C-Myc and myogenin. This study represents the first characterization step of the ZNF350 promoter. Additional studies in larger cohorts and other populations will be needed to further evaluate whether common and/or rare ZNF350 promoter variants and haplotypes could be associated with a modest risk of breast cancer.
\end{abstract}

Journal of Human Genetics (2013) 58, 59-66; doi:10.1038/jhg.2012.127; published online 15 November 2012

Keywords: breast cancer susceptibility; haplotypes; promoter; ZNF350 variants

\section{INTRODUCTION}

In 2011, breast cancer was the most common cancer not only in Canadian women, representing $28 \%$ of all new cancers and $14.4 \%$ of cancer death, but also in Western countries. ${ }^{1,2}$ In mid 1990s, the two major genes BRCA1/2 were identified as strongly associated with breast cancer susceptibility in high-risk breast cancer families. $^{3-5}$ Variations in several genes of lower penetrance/ frequency, such as TP53, PTEN, ATM, CHEK2, PALB2 and $B R I P 1$, are also associated with breast cancer risk but together with BRCA1/2, these genes would explain only $25 \%$ of the familial breast cancer risk. ${ }^{6} \mathrm{~A}$ significant portion of the unexplained cancer predisposition could be associated, among others, with variations in BRCA1-interacting partners resulting in reduction of BRCA1 activity and accumulation of mutations and alteration of the genome integrity. In addition to a key role in homologous recombination repair through its interactions with Rad51 and FANCD2, ${ }^{7-9}$ BRCA1 is also involved in cell cycle G2/M checkpoint by acting as a co-repressor of GADD45A (growth arrest and DNA damage gene 45) transcription in association with the zinc-finger protein $350\left(\right.$ ZNF350). ${ }^{10-12}$

ZNF350 protein, also known as ZBRK1 (zinc-finger and BRCA1interacting protein with a KRAB domain 1), has been shown to regulate the expression of many genes by binding the GGGxxx CAGxxxTTT consensus sequence. ${ }^{12}$ In particular, ZNF350 is a transcriptional repressor of GADD45A occurring in a BRCA1dependent manner, which involves a binding site in intron 3 of GADD45A gene. ${ }^{12}$ Moreover, given that ZNF350 DNA recognition motif sequences have been found in many BRCA1-targeted genes, a common function of ZNF350 in cellular DNA damage repair response has been suggested. ${ }^{12}$

ZNF350 has been shown to be involved in the tumorigenesis development of several human cancers. The under-expression of ZNF350 gene is observed in breast and colon carcinogenesis as well as in cervical tumor cells. ${ }^{13-15}$ Moreover, the inhibition of malignant growth, invasion and metastasis in cervical cells is correlated with high levels of ZNF350 gene, therefore suggesting a role of tumor

Cancer Genomics Laboratory, Oncology and Molecular Endocrinology Research Centre, Centre Hospitalier Universitaire de Québec and Laval University, Québec city, QC, Canada

Correspondence: Dr F Durocher, Cancer Genomics Laboratory, Oncology and Molecular Endocrinology Research Centre, Centre Hospitalier Universitaire de Québec and Laval University, 2705 Laurier Boulevard, T2-53, Québec city, QC, Canada G1V 4G2

E-mail: Francine.durocher@crchul.ulaval.ca

Received 15 June 2012; revised 2 October 2012; accepted 15 October 2012; published online 15 November 2012 
suppressor gene. ${ }^{15}$ This upregulation leads to the increased expression of several genes involved in gene expression, cellular growth and proliferation. $^{15}$

In particular, the co-repressor complex ZNF350/BRCA1/CtIP (CtTB-interacting protein) is implicated in the repression of highmobility group AT-hook 2 (HMGA2) and angiopoietin-1 (ANG1) genes, which are involved in increased proliferation, mammary acini formation, anchorage-independent growth and vascular formation in breast tumors. ${ }^{16,17}$ In addition, the ZNF350 gene overexpression led to an increase of ataxin-2 (ATXN2) mRNA levels (spinocerebellar ataxia type 2: SCA2 gene), ${ }^{18}$ which is involved in RNA metabolism and endocytic processes. ${ }^{19-23}$ In breast cancer cells, ZNF350 was also identified as a transcriptional repressor of p21 when associated with the KRAB domain-associated protein $1 .^{24,25}$ Furthermore, the expression of the ZNF350 gene may be repressed through an E2F1recognition sequence in its promoter region, which allows the binding of the RB/E2F1/CtIP/CtBP complex responsible for this repression. ${ }^{26}$ Deregulation of this repression leading to an increase of ZNF350 levels could result in cellular sensitivity of DNA damage and ultimatety in carcinogenesis.

Based on the potential association of ZNF350 gene variations with breast cancer susceptibility described previously ${ }^{27}$ and its role in DNA repair and carcinogenesis development as well as the importance of the fine regulation of ZNF350 gene expression described above, the analysis and characterization of promoter variants regulating the expression of the ZNF350 gene became of great interest. We therefore characterized the sequence variations in the ZNF350 promoter region and evaluated their association with breast cancer risk in the French Canadian population. To our knowledge, this is the first analysis of the ZNF350 gene promoter describing the effect of genomic variants on gene expression.

\section{MATERIALS AND METHODS}

\section{Ascertainment of families and genomic DNA extraction}

All 96 non-BRCA1/2 individuals from high-risk French Canadian breast and ovarian cancer families (that is, families in which multiple cases of breast/ovarian cancer are present in close relatives-three cases in first- or four cases in second-degree relatives-or with strong evidence of a familial component) participating in this study were originally part of a larger interdisciplinary program termed INHERIT BRCAs. ${ }^{28}$ All participants were at least 18 years of age, mentally capable and had to sign an informed consent form. Ethics committees reviewed the research project at the participating institutions from which the patients were referred. The details regarding selection criteria of the breast cancer cases as well as the experimental and clinical procedures have been described previously. ${ }^{28-30}$ A subset of 97 highrisk French Canadian breast/ovarian cancer families was drawn from the initial study based on the absence of detectable BRCA1/2 mutation (so-called BRCAX) and constituted the cohort used for another study specifically aiming at the identification of other susceptibility loci/genes to breast cancer. One individual affected with breast cancer per family was selected for analysis, with a selection preference for the youngest subject available in the family. In all instances, diagnosis of breast cancer was confirmed by pathology reports. Lymphocytes from breast cancer individuals were isolated and immortalized as previously described. ${ }^{27,31-33}$ Genomic DNA extraction of the 96 breast cancer cases as well as 94 healthy individuals from the same population has been performed as previously described. ${ }^{30}$ The healthy blood samples were obtained from Dr Damian Labuda at the Centre de Cancérologie Charles Bruneau, Hôpital Ste-Justine, Montreal, QC, Canada. The individuals who provided these samples were recruited on a nonnominative basis, in the framework of long-term studies aiming at the characterization of the genetic variability in human populations, approved by the Institutional Ethic Review Board.

\section{ZNF350 promoter sequencing, sequence analysis and variant} characterization

Based on the genomic position of the human ZNF350 gene (chromosome 19 GRCh37.p2; 52490079-52467593), the PCR amplification was performed on breast cancer cases and controls using primers designed to amplify the $2-\mathrm{kb}$ region upstream the ZNF350 gene with the primer pairs listed in Supplementary Table 1. Direct sequencing and sequence analysis were performed as described previously. ${ }^{30}$ The open-source toolset PLINK was used to determine the deviation from Hardy-Weinberg equilibrium (HWE), and to calculate Fisher's exact test and odds ratio with $95 \%$ confidence interval for each variant. Identification of potential transcription factor (TF)-binding sites were predicted using the MatInspector ${ }^{34}$ and Transcription Element Search softwares. ${ }^{35}$

\section{Haplotype and linkage disequilibrium (LD) block estimation}

To estimate the pattern of $\mathrm{LD}$, all 12 variations identified in our breast cancer case series have been genotyped. The LDA program ${ }^{36}$ was used to calculate pairwise LD for each SNP pair. Lewontin's $\left|D^{\prime}\right|$ measures were used to illustrate a graphical overview of LD between variants. ${ }^{36,37}$

LD block identification was performed with the variants having a minor allele frequency (MAF) $>2 \%$ using the Haploview software ${ }^{38}$ based on the algorithm of confidence intervals. Tagging SNPs (tSNP) from each LD block were then identified using the same software. The Caucasian HapMap data from the CEPH/CEU cohort was used to compare with the French Canadian population.

Haplotype analysis was performed using PHASE 2.1.1 software. ${ }^{39}$ The PHASE program estimates haplotype frequencies with a Bayesian-based algorithm and then uses a permutation test to determine the significance of differences in inferred haplotypes between both sample sets. All association tests were run under default conditions with 100000 permutations. Haplotype frequencies were estimated using the promoter and gene variants having a MAF $>2 \%$ (in at least one series).

\section{Luciferase promoter assays}

A 2410-bp fragment of the human ZNF350 promoter region including the untranslated exon 1 was PCR-amplified using genomic DNA from breast cancer individuals carrying the haplotypes $\mathrm{H} 4, \mathrm{H} 8, \mathrm{H} 10$ and $\mathrm{H} 12$ using primers introducing a XhoI or HindIII restriction site. The primers used for PCR were: 5'-GACGACCTCGAGGAGAAGCCCGAGCTAGGAAG-3' (XhoI) and $5^{\prime}$-GACGACAAGCTTGGCCGTTGATCACTACAGACCC-3' (HindIII) PCR products were then digested and introduced into the pGL3-basic vector (Promega Corporation, Madison, WI, USA). Single-promoter variant haplotypes (p1, p4, p5, p7, p11 or p12) were generated using the wild-type ZNF350 promoter haplotype $\mathrm{H} 8 /$ luciferase reporter construct as a template and the Quickchange II site-directed mutagenesis kit (Stratagene, La Jolla, CA, USA) according to the manufacturer's protocol. Following transformation and plasmids extraction, plasmid constructions integrity were confirmed by sequencing. Transient transfection in ZR-75-1 and MCF-7 cells and DualLuciferase Reporter assays were performed in five replicates. The human breast adenocarcinoma cell line MCF-7 was grown in DMEM/F12 (Wisent, St-Bruno, QC, Canada) supplemented with 5\% FBS, 1\% Penicillin-Streptomycin and E2 $10 \mathrm{~nm}$ to enhance cell growth. The human breast adenocarcinoma cell line ZR75-1 was maintained in RPMI1640 (Wisent) supplemented with 10\% FBS, $1 \%$ penicillin-streptomycin and E2 $10 \mathrm{~nm}$ to enhance cell growth. Cells were seeded in 24-well culture plate at a density of $50-70 \%$ and incubated overnight. Using ExGen500 according to the manufacturer's protocol (Fermentas Canada Inc., Burlington, ON, Canada), each well was transfected with $800 \mathrm{ng}$ of pGL3promoter haplotype-specific construct (or the empty pGL3 vector) encoding a modified firefly luciferase gene and co-transfected with $200 \mathrm{ng}$ of pRL-null vector (Promega) encoding the renilla luciferase gene as an internal standard. The pGL3-basic vector and pGL3-SV40 control vector were used as negative and positive controls, respectively. Following a 24-h incubation, cells were assayed for the luciferase reporter gene activities measured with the DualLuciferase Reporter Assay System according to the manufacturer's instructions (Promega) in a MicroLumat Plus luminometer (EG\&G Berthold, Bad Wildbad, Germany). Promoter activities were expressed as a ratio of firefly 
luciferase to renilla luciferase luminescence in each well. The empty pGL3-basic vector was used to measure basal expression levels in each cell line.

\section{RESULTS}

Direct sequencing of the ZNF350 promoter region in 96 BRCA1/2negative breast cancer subjects from high-risk French Canadian breast/ ovarian cancer families and 94 healthy controls led to the identification of 12 variants. Among the promoter genomic variants identified, five of them, namely c. $-1775 \mathrm{~T}>\mathrm{A}, \quad$ c. $-1769 \mathrm{~T}>\mathrm{A}, \quad$ c.-895delATCA, c. $-873 \mathrm{C}>\mathrm{T}$ and c.-856insAA are novel variations not reported in databases. As shown in Table 1, 5 out of the 12 variants display a MAF lower than $2 \%$ in either of the series. The variants c. $-1775 \mathrm{~T}>\mathrm{A}, \mathrm{c}$.$1769 \mathrm{~T}>\mathrm{A}$, c. $-872 \mathrm{G}>\mathrm{C}$ and c.-856insAA are found exclusively in the control group, whereas the c.895delATCA is observed in one breast cancer case at the heterozygous state. Only the c.-874 G > A variant displays a significant deviation from HWE due to an excess of homozygotes (HWE $P=0.032$ ) among the healthy individuals.

To combine genotype data from the promoter region and those from the ZNF350 gene published by Desjardins et al. ${ }^{27}$ for LD and haplotype analyses, a subset of 67 healthy controls, which were commonly genotyped in the previous and current publication was selected. In addition, the ZNF350 gene variants from Desjardins et $a .^{27}$ having a MAF $>2 \%$ have also been included in Table 1 as reference for LD and haplotype analyses (denoted g2 to g17). Considering all sequence variations, the c. $425 \mathrm{~T}>\mathrm{C}, \mathrm{c} .466+46 \mathrm{~A}>\mathrm{T}$, c. $936 \mathrm{C}>\mathrm{T}$ and c.1900C/T variants located in the gene region displayed a modest significant protection against breast cancer, whereas none of the promoter variants showed any significant difference of MAF between both series.

A graphical representation of the pairwise LD between the 18 ZNF350 promoter and gene variants having a MAF $>2 \%$ in at least one series, as measured by Lewontin's $\left|D^{\prime}\right|$ values, is shown in Supplementary Figure 1. As demonstrated, the majority of the genomic variations are in strong $\mathrm{LD}$ with each other. Although the ZNF350 gene is comprised in a relative short genomic region, strong LD was found between the two most distantly separated promoter and gene variants ( $\mathrm{p} 1$ and g17: inter-marker distance $\sim 25 \mathrm{~kb}$, $\left.\left|D^{\prime}\right|=0.95\right)$, which suggested that LD at the ZNF350 locus did not decrease significantly with distance. However, few intragenic variants showed lower LD with other variations, namely g10 and g11, located in the coding region of the gene, whereas a clear breakage of LD seemed to occur between the promoter and gene regions, excepting for g2, which demonstrated a strong LD with all other promoter variants.

PHASE analyses identified 13 different haplotypes in the promoter region of ZNF350 in breast cancer and control individuals (Table 2a). According to PHASE, the promoter haplotype H8 was the major haplotype with a frequency of $80.6 \%$ in both series combined. The four most frequent haplotypes (H4, H8, H11 and H12) represent $91.8 \%$ of all haplotypes identified. The H1, H3 and $\mathrm{H} 10$ haplotypes were found exclusively in breast cancer cases, whereas four haplotypes were unique to the control group $(\mathrm{H} 2, \mathrm{H} 6, \mathrm{H} 9$ and $\mathrm{H} 13)$. No significant difference of global haplotype frequencies was identified between both series $(P=0.619)$. However, PHASE analyses performed with both series including the variants from both the promoter and the gene regions (with MAF $>2 \%$ ) revealed a strong significant difference with a $P$-value of 0.00092 (Table 2b). The haplotypes Hpg1 and Hpg33 were significantly over-represented in breast cancer cases, having $P$-values of 0.036 and 0.011 , respectively.

The identification of tSNPs was then carried out in two subsequent steps, firstly by determining haplotype blocks, followed by the identification of tSNPs in each LD block. Based on the algorithm from Gabriel et al., ${ }^{40}$ three LD blocks encompassing the ZNF350 gene have been identified in the French Canadians by the Haploview software (expectation maximization algorithm) (Figure 1). To confirm the reliability of our data, HapMap data (from caucasian population) have also been analyzed and although using a different panel of SNPs, three LD blocks were also identified. The composition and regions of recombination of the three blocks was relatively similar between the French Canadian and CEPH/CEU data sets. The promoter region is included in the first LD block, whereas the gene region is divided in two LD blocks. Thereafter, considering haplotypes having a frequency $\geqslant 1 \%$, seven tSNPs were identified in the three LD blocks, namely variants p4, p5 and p11 found in block 1, g2 and g4 in block 2, whereas block 3 consists of variants g10 and g11.

As shown in Supplementary Table 2, in silico analysis using MatInspector and Transcription Element Search software indicated that the variant c.-1779 $\mathrm{T}$ led to the creation of a new binding site for two octamer-binding proteins (POU5F1, POU3F3), an autoimmune regulatory element binding factor (AIRE) as well as neurofibromin 1 (NF-1). Several new binding sites are generated by the variant c.-1171C namely for SOX-9, CART-1, Delta factor, GATA-1 and NF$\mathrm{E}$, whereas binding element sequences for the heat shock factor HSF2 and zinc-finger transcriptional repressor ZNF217 are abolished. As for the variant c.-922C, binding sites for the glucocorticoid receptor and GKLF are created. Interestingly, the c.-874A variant abolished binding sites for TFs involved in transcription such as E-box binding factors and RNA polymerase II TFIIB and created new binding elements for c-myc myelocytomatosis viral oncogene homolog (c-Myc) and myogenin. In particular, the c.-201 G variant generated a binding site for the MAF and AP1 related factors (AP1R). Finally, c.-85G resulted in new binding sites for the cellular and viral myb-like transcriptional regulators (MYBL) and abolished a Sp1 site.

To analyze the effect of promoter variants on ZNF350 transcription, the four promoter haplotypes showing the highest frequency namely H4, H8, H11 and H12, were analyzed using luciferase assays in the ZR-75-1 and MCF-7 breast cancer cell lines. The haplotypes that were present exclusively in one or two breast cancer cases $(\mathrm{H} 1$, $\mathrm{H} 3$ and H10) were not used in the analysis because they are under represented in the analyzed population (Table 2a). To discriminate the individual effect of each variant on transcription, single-variant haplotypes were generated by directed mutagenesis. The commonest haplotype $\mathrm{H} 8$ was used as reference for statistical analysis. $\mathrm{H} 4 \mathrm{did}$ not induce any significant difference of transcriptional activity, whereas the haplotypes H11 and $\mathrm{H} 12$ increased significantly the expression of the luciferase gene in both cell lines (Figure 2). Compared with the common haplotype $\mathrm{H} 8$, the only difference with haplotype $\mathrm{H} 11$, which revealed a significant higher luciferase activity (by more than 2.5 fold on its own), resides in the presence of the variant c. $-874 \mathrm{G}>\mathrm{A}$ variant (p7).

As for the haplotype H12, it is composed of multiple variants not found in $\mathrm{H} 8$, namely $\mathrm{p} 1, \mathrm{p} 4, \mathrm{p} 5, \mathrm{p} 8$ and $\mathrm{p} 12$ and showed also a significant increase of luciferase expression. In addition, each single variant on its own led also to significant increased expression of luciferase activity.

\section{DISCUSSION}

The vast majority of genes identified so far and showing a high/ moderate penetrance in breast cancer susceptibility are directly involved in DNA repair mechanisms and cell cycle control (BRCA1, BRCA2, RAD51C, PALB2, BRIP1, TP53, PTEN, ATM and CHEK2), ${ }^{41-45}$ therefore proteins involved in these mechanisms and/or interacting 
Table 1 Sequence variations in ZNF350 gene and genotype frequencies in familial breast cancer cases and controls

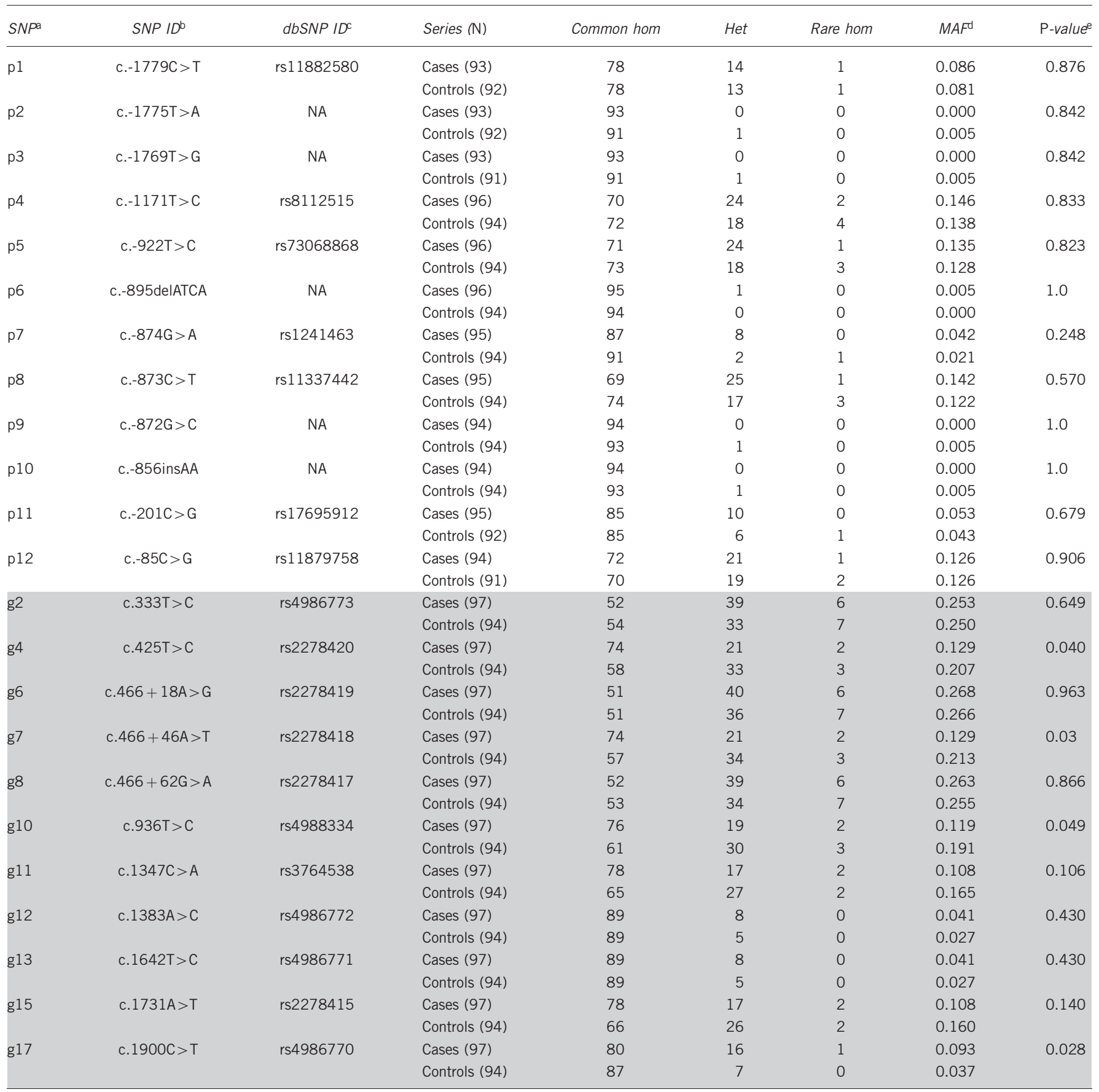

Abbreviation: SNP, single-nucleotide polymorphism.

Grey shading corresponds to gene variants identified in Desjardins et al. ${ }^{27}$

${ }^{\text {ap}}$, Promoter variants; g, gene variants (MAF $>2 \%$ ) according to Table 1 included in Desjardins et al. ${ }^{27}$

bSNP ID are indicated according to the nomenclature guidelines of the Human Genome Variation Society. The first base from the mRNA RefSeg NM 021632.2 is designated as +1. The ZNF350 genomic sequence used for promoter SNP reference corresponds to the chromosome 19 GRCh37.p2; 52492179-52467593 of UCSC, with the mRNA + 1.

cdbSNP ID is indicated according to build 135; NA: SNP not found in the database.

dMAF, minor allele frequency.

e $P$-value (Fisher) of the minor allele versus the common allele.

with BRCA1 or BRCA2, such as ZNF350, represent excellent candidate genes to be studied regarding their potential implication in breast cancer predisposition. The 96 non-BRCA1/2 breast cancer cases included in our cohort have been selected based on their strong family history and come from 96 high-risk French Canadian breast and ovarian cancer families displaying multiple individuals affected with breast cancer. This study design has been demonstrated to substantially decrease the number of cases and controls to achieve the same magnitude of power compared with studies based solely on breast cancer cases unselected for family history. ${ }^{46}$

In a previous analysis, we identified a potential association of genomic variations located in the $5^{\prime}$-part of the ZNF350 gene with breast cancer predisposition. ${ }^{27}$ However, as described in the previous section, further analyses of the variants located within the promoter 
Table 2 (a) Estimated haplotype frequencies of ZNF350 gene using promoter variants having a frequency $>2 \%$ in the breast cancer case and control series ${ }^{\mathrm{a}}$. (b) Frequencies of estimated haplotypes of ZNF350 gene using promoter and gene variants $(\mathrm{Hpg})$ having a frequency $>2 \%$ in the breast cancer case and control series ${ }^{b}$

\begin{tabular}{llccc} 
(a) & & Frequency & Frequency & \multicolumn{1}{c}{ P- } \\
(cases) & (controls) & value \\
\# Hap & p1-4-5-7-8-11-12 & 0.005 & 0.000 & 0.495 \\
\hline H1 & TCTGCCC & 0.000 & 0.005 & 0.495 \\
H2 & CCCGTCG & 0.005 & 0.000 & 0.495 \\
H3 & CCCGTGC & 0.047 & 0.032 & 0.600 \\
H4 & CCCGTGG & 0.010 & 0.016 & 0.683 \\
H5 & CCTGCCC & 0.000 & 0.005 & 0.495 \\
H6 & CCTGTGG & 0.005 & 0.011 & 0.620 \\
H7 & CTCGCCC & 0.800 & 0.819 & 0.604 \\
H8 & CTTGCCC & 0.000 & 0.010 & 0.244 \\
H9 & CTTGCCG & 0.010 & 0.000 & 0.499 \\
H10 & CTTGTCC & 0.042 & 0.021 & 0.380 \\
H11 & CTTACCC & 0.078 & 0.0741 & 1.0 \\
H12 & TCCGTCG & 0.000 & 0.005 & 0.495 \\
H13 & TCCGTGC & & &
\end{tabular}

(b)

\begin{tabular}{|c|c|c|c|c|}
\hline \# Hap & $\begin{array}{l}p 1-4-5-7-8-11-12-g 2-4-6-7-8- \\
10-11-12-13-15-17^{c}\end{array}$ & $\begin{array}{l}\text { Frequency } \\
\text { (cases) }\end{array}$ & $\begin{array}{l}\text { Frequency } \\
\text { (controls) }\end{array}$ & $\begin{array}{l}\mathrm{P}- \\
\text { value }^{\mathrm{d}}\end{array}$ \\
\hline Hpg1 & CTTGCCCTTAAGTCATAC & 0.682 & 0.567 & 0.036 \\
\hline Hpg2 & CTTGCCCTTAAGTCATAT & 0.005 & 0.007 & 0.514 \\
\hline Hpg3 & CTTGCCCTTAAGCCATAC & 0.000 & 0.015 & 0.168 \\
\hline Hpg4 & CTTGCCCTTAAGCCATTC & 0.000 & 0.007 & 0.411 \\
\hline Hpg5 & CTTGCCCTTAAGCAATAC & 0.000 & 0.015 & 0.168 \\
\hline Hpg6 & CTTGCCCTTAAGCAATTC & 0.000 & 0.015 & 0.168 \\
\hline Hpg7 & CTTGCCСTTAAACAATTC & 0.000 & 0.007 & 0.411 \\
\hline Hpg8 & CTTGCCCTTATGTCATAC & 0.000 & 0.007 & 0.411 \\
\hline Hpg9 & CTTGCCCTTGAGTCATAC & 0.005 & 0.015 & 0.570 \\
\hline Hpg10 & CTTGCCCTTGTGTCATAC & 0.005 & 0.022 & 0.309 \\
\hline Hpg11 & CTTGCCCTCAAGCAATTC & 0.000 & 0.007 & 0.411 \\
\hline Hpg12 & CTTGCCССTGAATCATAC & 0.000 & 0.007 & 0.411 \\
\hline Hpg13 & СTTGCCCCTGAATCATAT & 0.005 & 0.000 & 1.0 \\
\hline Hpg14 & CTTGCCСССАAАCAАTTC & 0.000 & 0.007 & 0.411 \\
\hline Hpg15 & CTTGCCCCCATACAATTC & 0.000 & 0.007 & 0.411 \\
\hline Hpg16 & CTTGCCCCCGAATCATAC & 0.000 & 0.007 & 0.411 \\
\hline Hpg17 & CTTGCCCCCGTATCATAC & 0.000 & 0.037 & 0.011 \\
\hline Hpg18 & CTTGCCCCCGTATAATTC & 0.000 & 0.007 & 0.411 \\
\hline Hpg19 & CTTGCCCCCGTACAATTC & 0.099 & 0.097 & 1.0 \\
\hline Hpg20 & CTTGTCCTTAAGTCATAC & 0.010 & 0.000 & 0.514 \\
\hline Hpg21 & СTTACCCTTAAGTCATAC & 0.042 & 0.015 & 0.207 \\
\hline Hpg22 & CTCGCCCCCGTACAATTC & 0.005 & 0.007 & 0.514 \\
\hline Hpg23 & СCTGCCCCCGTACCATAC & 0.010 & 0.015 & 0.646 \\
\hline Hpg24 & СCTGTGGCTGAATCCCAC & 0.000 & 0.007 & 0.411 \\
\hline Hpg25 & CCCGTCGTTAAGTCATAC & 0.000 & 0.007 & 0.411 \\
\hline Hpg26 & CCCGTGCCTGAATCCCAC & 0.005 & 0.000 & 1.0 \\
\hline Hpg27 & CCCGTGGTTAAGTCATAC & 0.000 & 0.007 & 0.411 \\
\hline Hpg28 & CCCGTGGCTGAATCCCAC & 0.036 & 0.022 & 0.534 \\
\hline Hpg29 & CCCGTGGCCGTATCATAC & 0.010 & 0.015 & 0.646 \\
\hline Hpg30 & TCTGCCCCTGAATCATAT & 0.005 & 0.000 & 1.0 \\
\hline Hpg31 & TCCGTCGTTAAGTCATAT & 0.000 & 0.022 & 0.068 \\
\hline Hpg32 & TCCGTCGTTAAGCAATTC & 0.000 & 0.007 & 0.411 \\
\hline Hpg33 & TCCGTCGCTGAATCATAT & 0.078 & 0.015 & 0.011 \\
\hline
\end{tabular}

aGlobal Fisher's $P$-value between both series: 0.619 .

GGlobal Fisher's $P$-value between both series: 0.00092 .

'According to Table 1.

dFisher's $P$-value. region $(\sim 2 \mathrm{~kb})$ revealed no significant association of these variants with breast cancer susceptibility based on their MAF observed in breast cancer and control individuals. Moreover, haplotype analyses using exclusively the promoter variants support this observation. Nonetheless, when using a combination of promoter and gene variants for haplotype prediction, the analyses revealed a potential significant over-representation of Hpg1 and Hpg33 in breast cancer cases. Hpg1 is considered the common allele, whereas Hpg33 is characterized particularly by the presence of several nucleotide changes, such as the p12 (c.-85C>G) and g17 (c.1900C > T) variants (Table 1 and Figure 2b). Of interest, p12 is the closest promoter variant of the $5^{\prime}$-UTR region of the ZNF350 gene, which supports the association described previously. ${ }^{27}$

As seen in Figure 2, determination of the haplotype blocks including promoter and gene variants clearly identified a strong LD breakage between the promoter and gene regions. This LD breakage could explain the absence of breast cancer association for the promoter variants in contrast to previous results regarding the involvement of gene variants located in the 5'portion of the ZNF350 gene. ${ }^{27}$ Moreover, the $\mathrm{g} 2$ and g4 variants have been identified as tSNPs by the Haploview program. Although the $D^{\prime}$ value observed between p12 and g2 was high (Supplementary Figure 1) and did not confirm the first LD breakage observed between the promoter and gene regions as illustrated in Figure 1, the second LD breakage predicted in the vicinity of g8 and g10 variants is confirmed by the low $D^{\prime}$ values associated with both variants.

Considering that the expression of the ZNF350 gene is crucial for cell cycle control and that this expression has been reported to be altered in cancer, we evaluated the impact of promoter variations on gene expression using luciferase assays. Given that p4-5-8-11-12 are included in $\mathrm{H} 4$ and that this haplotype did not trigger any significant modulation of transcriptional activity, we can conclude that $\mathrm{p} 1$ and $\mathrm{p} 7$ are likely the variants responsible for the upregulation of luciferase expression. As illustrated in Figure 2, each single variant studied ( $1, \mathrm{p} 4, \mathrm{p} 5, \mathrm{p} 7, \mathrm{p} 8, \mathrm{p} 11$ and $\mathrm{p} 12)$ possesses the capacity on its own to increase the transcriptional activity of the ZNF350 gene promoter, with the c.-874G $>$ A variant producing the highest increase in expression. However one has to keep in mind that obviously a complex combination of variants is likely involved in the specific expression of each observed haplotype.

In the same line of thoughts, regarding the TFs potentially involved in the modulation of transcriptional activity related to the presence of the p1 (c.-1779C $>\mathrm{T}$ ) and p7 (c.-874G $>\mathrm{A}$ ) variants specifically, the c.-1779 $\mathrm{T}$ variation leads to the creation of a new binding site for POU5F1 (OCT3/4), whereas the c.-874A abolished binding elements for MYC-MAX and TFIIB and creates a new binding sequence for c-Myc. The octamer-binding protein POU5F1 is known to control pluripotency of embryonic stem cells and is required for the initial formation of a pluripotent founder cell population in the mammalian embryo. ${ }^{47}$ POU5F1 is a member of the POU family of transcriptional activators, which control the expression of its target genes through binding of an AGTCAAAT consensus motif sequence. ${ }^{48,49}$ Of interest, POU5F1 has been shown to be expressed exclusively in human breast cancer cells, being not detected in normal breast tissue. In addition, the overexpression of this TF has been demonstrated to induce the expression of the endogeneous fibroblast growth factor-4 gene in human breast cancer cells. ${ }^{50}$ The potential activation of ZNF350 gene expression potentially triggered by the binding of the POU5F1 protein to its promoter is in accordance with the predicted cell proliferation following POU5F1-binding effect 
a

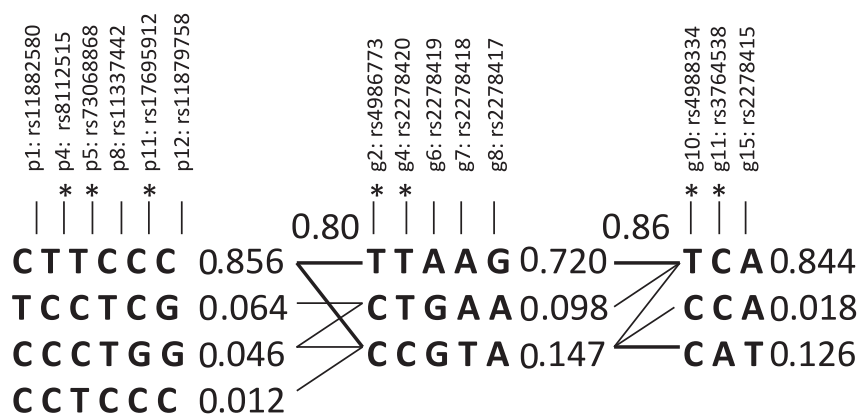

b

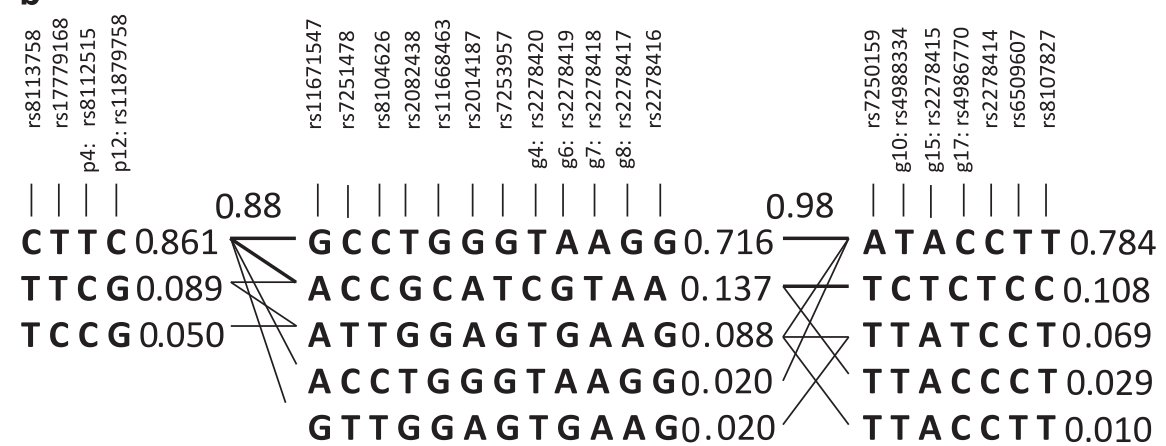

Figure 1 Haplotype blocks and tSNPs identified in the ZNF350 gene. (a) Predicted haplotype blocks using promoter (p) and genomic (g) variants identified in the case series showing a MAF higher than 2\% (18 variants) in the French Canadian population. (b) Predicted haplotype blocks using HapMap data from CEPH/CEU cohort. The distance between the variants are similar than in (a). tSNPs identified on a block-by-block basis are denoted with an asterisk (*) above the variation number and have been selected based on haplotypes showing a frequency higher than $1 \%$. Population haplotype frequencies are displayed on the right of each haplotype combination while the level of recombination is displayed above the connections between two blocks. Thick connections represent haplotypes with frequencies higher than 10\%, whereas frequencies below $10 \%$ are represented by thin lines.

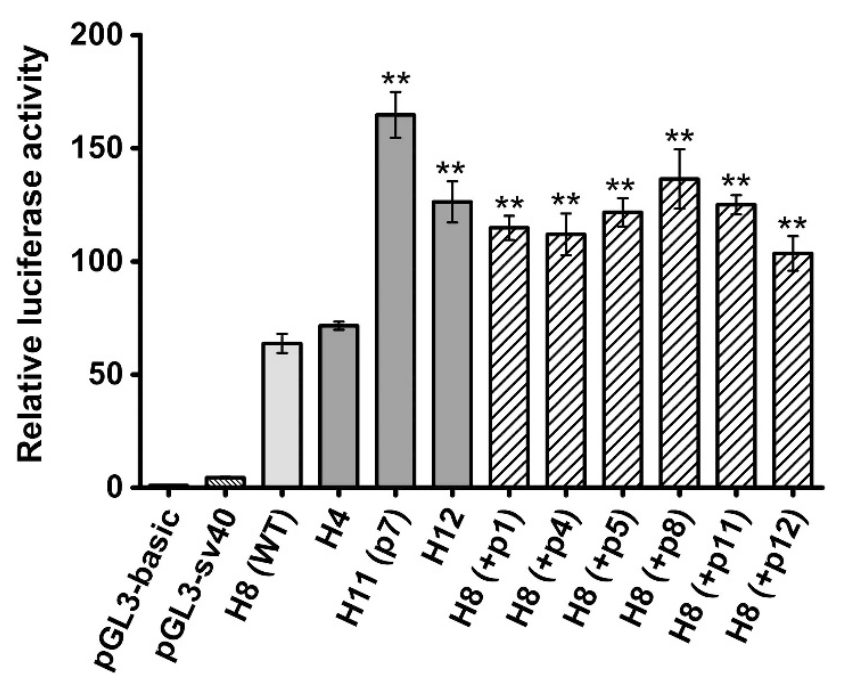

Figure 2 Luciferase assays. Effect of multiple promoter variants on ZNF350 gene promoter activity using luciferase reporter assay. ZR-75-1 cells were transiently co-transfected with the Renilla reporter plasmid (pRL) as a transfection control. Each data represents mean \pm s.d. of five replicates. Data are shown as relative induction compared with the activity of cells transfected with the empty pGL3-basic luciferase reporter vector. $(* * P<0.01)$. combined with the increased repression (caused by the increased expression of ZNF350) of the GADD45A protein, which represents a growth arrest-associated gene. ${ }^{12}$

As for TFIIB, it is involved in start site selection, promoter binding and promoter bending during initiation. This protein is a component of the set of basal TFs required to allow specific binding of the RNA polymerase II protein on promoter sequences, ${ }^{51,52}$ whereas MYCMAX dimer has been demonstrated to activate transcription of reporter genes in an E-box-dependent manner. ${ }^{53-55}$ Despite the suppression of binding sites for TFs known to activate gene transcription such as MYC-MAX and TFIIB, it seems that the creation of a c-Myc-binding site by the c.-874 G > A variant could overturn the disruption of the potential promoter-binding element of MYC-MAX and TFIB, known to stimulate transcription. Indeed in addition to its heterodimerization with MAX, the MYC protein could also form homodimers to bind DNA. ${ }^{56,57}$ Moreover, MYC functions independent of MAX, such as the regulation of Pol III, have recently been demonstrated in a Drosophila model. ${ }^{58}$ Altogether, this supports the potential action of MYC protein (without MAX) in the regulation of transcriptional activity of ZNF350 gene. Hence the creation of a new binding site for the c-Myc protein could be responsible for the upregulation of transcriptional activity observed in the presence of the c. $-874 \mathrm{~A}$ variation.

This study represents the first description of genomic variants influence at the promoter level of the ZNF350 gene, and the 
information is still very limited and scarce regarding the characterization of the ZNF350 gene in relation with breast cancer. Low levels of ZNF350 have been observed in tumor tissue, but on the other hand an increase of expression of ZNF350 is associated, together with BRCA1, with a repression of GADD45A, and could subsequently lead to an increase of DNA damage and carcinogenesis because the low expression of GADD45A could not induce cell cycle arrest. Taking this into account, it is tempting to speculate that the increase of ZNF350 expression triggered by the promoter sequence variations described herein could be involved in tumorigenesis initiation rather than in tumorigenesis development. However, this would have to be further tested in additional studies.

\section{CONFLICT OF INTEREST}

The authors declare no conflict of interest.

\section{ACKNOWLEDGEMENTS}

We thank all individuals and families who participated in this study. We thank M Tranchant, Dr M Dumont and G Leblanc of the Cancer Genomics Laboratory for sample management and mutation screening. We also thank Dr D Labuda and C Moreau at the Centre de Cancérologie Charles Bruneau of Ste-Justine Hospital for help with control DNA samples. We also acknowledge the work of Professor Bartha Maria Knoppers and her colleagues regarding ELSI issues, as well as the ethics committees of all participating institutions. This work was supported by the Canadian Institutes of Health Research (CIHR) and Institute of Cancer and Institute of Gender and Health for the INHERIT BRCAs research program, the Fond de la Recherche en Santé du Québec (FRSQ)/Réseau de Médecine Génétique Appliquée (RMGA), the Canadian Breast Cancer Research Alliance and the CURE Foundation. SD held studentships from Fondation René Bussières and Fondation Desjardins, and FD was recipient of a chercheur-boursier from the FRSQ and a Research Career Award in the Health Sciences from CIHR/R\&D Health Research Foundation.

1 World Health Organization. World Health Statistics 2012. http://www.who.int/gho/ publications/world_health_statistics/EN_WHS2012_Full.pdf.

2 Cancer Research Society. http://www.cancer.ca/canada-wide.aspx.

3 Tavtigian, S. V., Simard, J., Rommens, J., Couch, F., Shattuck-Eidens, D., Neuhausen, $\mathrm{S}$. et al. The complete BRCA2 gene and mutations in chromosome 13q-linked kindreds. Nat. Genet. 12, 333-337 (1996).

4 Wooster, R., Bignell, G., Lancaster, J., Swift, S., Seal, S., Mangion, J. et al. Identification of the breast cancer susceptibility gene BRCA2. Nature 378, 789-792 (1995).

5 Miki, Y., Swensen, J., Shattuck-Eidens, D., Futreal, P. A., Harshman, K., Tavtigian, S. et al. A strong candidate for the breast and ovarian cancer susceptibility gene BRCA1. Science 266, 66-71 (1994).

6 Stratton, M. R. \& Rahman, N. The emerging landscape of breast cancer susceptibility. Nat. Genet. 40, 17-22 (2008).

7 Scully, R., Chen, J., Plug, A., Xiao, Y., Weaver, D., Feunteun, J. et al. Association of BRCA1 with Rad51 in mitotic and meiotic cells. Cell 88, 265-275 (1997).

8 Jin, Y., Xu, X. L., Yang, M. C., Wei, F., Ayi, T. C., Bowcock, A. M. et al. Cell cycledependent colocalization of BARD1 and BRCA1 proteins in discrete nuclear domains. Proc. Natl Acad. Sci. USA 94, 12075-12080 (1997).

9 Wang, Y., Cortez, D., Yazdi, P., Neff, N., Elledge, S. J. \& Qin, J. BASC a super complex of BRCA1-associated proteins involved in the recognition and repair of aberrant DNA structures. Genes Dev. 14, 927-939 (2000).

10 Fan, W., Jin, S., Tong, T., Zhao, H., Fan, F., Antinore, M. J. et al. BRCA1 regulates GADD 45 through its interactions with the OCT-1 and CAAT motifs. J. Biol. Chem. 277, 8061-8067 (2002).

11 Mullan, P. B., Quinn, J. E., Gilmore, P. M., McWilliams, S., Andrews, H., Gervin, C. et al. BRCA1 and GADD45 mediated G2/M cell cycle arrest in response to antimicrotubule agents. Oncogene 20, 6123-6131 (2001).

12 Zheng, L., Pan, H., Li, S., Flesken-Nikitin, A., Chen, P. L., Boyer, T. G. et al. Sequencespecific transcriptional corepressor function for BRCA1 through a novel zinc finger protein, ZBRK1. Mol. Cell 6, 757-768 (2000).

13 Garcia, V., Domnguez, G., Garcia, J. M., Silva, J., Peña, C., Silva, J. M. et al. Altered expression of the ZBRK1 gene in human breast carcinomas. J. Pathol. 202, 224-232 (2004).

14 Garcia, V., Garcia, J. M., Peña, C., Silva, J., Domnguez, G., Rodriguez, R. et al. The GADD45, ZBRK1 and BRCA1 pathway: quantitative analysis of mRNA expression in colon carcinomas. J. Pathol. 206, 92-99 (2005).
15 Lin, L. F., Chuang, C. H., Li, C. F., Liao, C. C., Cheng, C. P., Cheng, T. L. et al. ZBRK1 acts as a metastatic suppressor by directly regulating MMP9 in cervical cancer. Cancer Res. 70, 192-201 (2010).

16 Furuta, S., Wang, J. M., Wei, S., Jeng, Y. M., Jiang, X., Gu, B. et al. Removal of BRCA1/ CtIP/ZBRK1 repressor complex on ANG1 promoter leads to accelerated mammary tumor growth contributed by prominent vasculature. Cancer Cell 10, 13-24 (2006).

17 Ahmed, K. M., Tsai, C. Y. \& Lee, W. H. Derepression of HMGA2 via removal of ZBRK1/ BRCA1/CtIP complex enhances mammary tumorigenesis. J. Biol. Chem. 285, 44644471 (2010).

18 Hallen, L., Klein, H., Stoschek, C., Wehrmeyer, S., Nonhoff, U., Ralser, M. et al. The KRAB-containing zinc-finger transcriptional regulator ZBRK1 activates SCA2 gene transcription through direct interaction with its gene product, ataxin-2. Hum. Mol. Genet. 20, 104-114 (2011).

19 Lastres-Becker, I., Rüb, U. \& Auburger, G. Spinocerebellar ataxia 2 (SCA2). Cerebellum 7, 115-124 (2008).

20 Nonhoff, U., Ralser, M., Welzel, F., Piccini, I., Balzereit, D., Yaspo, M. L. et al. Ataxin-2 interacts with the DEAD/H-box RNA helicase DDX6 and interferes with P-bodies and stress granules. Mol. Biol. Cell 18, 1385-1396 (2007).

21 Nonis, D., Schmidt, M. H., van de Loo, S., Eich, F., Dikic, I., Nowock, J. et al. Ataxin-2 associates with the endocytosis complex and affects EGF receptor trafficking. Cell Signal. 20, 1725-1739 (2008).

22 Ralser, M., Albrecht, M., Nonhoff, U., Lengauer, T., Lehrach, H. \& Krobitsch, S. An integrative approach to gain insights into the cellular function of human ataxin-2. J. Mol. Biol. 346, 203-214 (2005).

23 Ralser, M., Nonhoff, U., Albrecht, M., Lengauer, T., Wanker, E. E., Lehrach, H. et al. Ataxin-2 and huntingtin interact with endophilin-A complexes to function in plastinassociated pathways. Hum. Mol. Genet. 14, 2893-2909 (2005).

24 Lee, Y. K., Thomas, S. N., Yang, A. J. \& Ann, D. K. Doxorubicin down-regulates Kruppel-associated box domain-associated protein 1 sumoylation that relieves its transcription repression on $\mathrm{p} 21^{\mathrm{WAF} 1 / \mathrm{CIP} 1}$ in breast cancer MCF-7 cells. J. Biol. Chem. 282, 1595-1606 (2007).

25 Madison, D. L. \& Lundblad, J. R. C-terminal binding protein and poly(ADP)ribose polymerase 1 contribute to repression of the p21 waf1/cip1 promoter. Oncogene 29, 6027-6039 (2010).

26 Liao, C. C., Tsai, C. Y., Chang, W. C., Lee, W. H. \& Wang, J. M. RB.E2F1 complex mediates DNA damage responses through transcriptional regulation of ZBRK1. J. Biol. Chem. 285, 33134-33143 (2010).

27 Desjardins, S., Belleau, P., Labrie, Y., Ouellette, G., Bessette, P., Chiquette, J. et al. Genetic variants and haplotype analyses of the ZBRK1/ZNF350 gene in high-risk non BRCA1/2 French Canadian breast and ovarian cancer families. Int. J. Cancer 122, 108-116 (2008).

28 Simard, J., Dumont, M., Moisan, A. M., Gaborieau, V., Malouin, H., Durocher, F. et al. Evaluation of $B R C A 1$ and $B R C A 2$ mutation prevalence, risk prediction models and a multistep testing approach in French-Canadian families with high risk of breast and ovarian cancer. J. Med. Genet. 44, 107-121 (2007).

29 Antoniou, A. C. \& Easton, D. F. Models of genetic susceptibility to breast cancer. Oncogene 25, 5898-5905 (2006).

30 Durocher, F., Labrie, Y., Soucy, P., Sinilnikova, O., Labuda, D., Bessette, P. et al. Mutation analysis and characterization of ATR sequence variants in breast cancer cases from high-risk French Canadian breast/ovarian cancer families. BMC Cancer 6, 230 (2006).

31 Guénard, F., Labrie, Y., Ouellette, G., Joly Beauparlant, C., Simard, J. et al. INHERIT BRCAs. Mutational analysis of the breast cancer susceptibility gene BRIP1/BACH1/ FANCJ in high-risk non-BRCA1/BRCA2 breast cancer families. J. Hum. Genet. 53, 579-591 (2008).

32 Durocher, F., Labrie, Y., Ouellette, G., BRCAs, I. N. H. E. R. I. T. \& Simard, J. Genetic sequence variations and $A D P R T$ haplotype analysis in French Canadian families with high risk of breast cancer. J. Hum. Genet. 52, 963-977 (2007).

33 Desjardins, S., Ouellette, G., Labrie, Y., Simard, J., BRCAs, I. N. H. E. R. I. T. \& Durocher, F. Analysis of GADD45A sequence variations in French Canadian families with high risk of breast cancer. J. Hum. Genet. 53, 490-498 (2008).

34 Quandt, K., Frech, K., Karas, H., Wingender, E. \& Werner, T. MatInd and MatInspector: new fast and versatile tools for detection of consensus matches in nucleotide sequence data. Nucleic Acids Res. 23, 4878-4884 (1995).

35 Schug, J. in Current Protocols in Bioinformatics (ed. Baxevanis, A. D.) 2.6.1-2.6.15 (John Wiley and Sons Inc, Hoboken, NJ, USA, 2003).

36 Ding, K., Zhou, K., He, F. \& Shen, Y. LDA-a java-based linkage disequilibrium analyzer. Bioinformatics 19, 2147-2148 (2003).

37 Devlin, B. \& Risch, N. A comparison of linkage disequilibrium measures for fine-scale mapping. Genomics 29, 311-322 (1995).

38 Barrett, J. C., Fry, B., Maller, J. \& Daly, M. J. Haploview: analysis and visualization of LD and haplotype maps. Bioinformatics 21, 263-265 (2005).

39 Stephens, M., Smith, N. J. \& Donnelly, P. A new statistical method for haplotype reconstruction from population data. Am. J. Hum. Genet. 68, 978-989 (2001).

40 Gabriel, S. B., Schaffner, S. F., Nguyen, H., Moore, J. M., Roy, J., Blumenstiel, B. et al. The structure of haplotype blocks in the human genome. Science 296, 2225-2229 (2002).

41 Meindl, A., Ditsch, N., Kast, K., Rhiem, K. \& Schmutzler, R. K. Hereditary breast and ovarian cancer: new genes, new treatments, new concepts. Dtsch Arztebl Int. 108, 323-330 (2011).

42 Meindl, A., Hellebrand, H., Wiek, C., Erven, V., Wappenschmidt, B., Niederacher, D. et al. Germline mutations in breast and ovarian cancer pedigrees establish RAD51C as a human cancer susceptibility gene. Nat. Genet. 42, 410-414 (2010). 
43 Turnbull, C. \& Rahman, N. Genetic predisposition to breast cancer: past, present, and future. Annu. Rev. Genomics Hum. Genet. 9, 321-345 (2008).

44 Antoniou, A., Pharoah, P. D., Narod, S., Risch, H. A., Eyfjord, J. E., Hopper, J. L. et al. Average risks of breast and ovarian cancer associated with $B R C A 1$ or $B R C A 2$ mutations detected in case series unselected for family history: a combined analysis of 22 studies. Am. J. Hum. Genet. 72, 1117-1130 (2003).

45 Ripperger, T., Gadzicki, D., Meindl, A. \& Schlegelberger, B. Breast cancer susceptibility: current knowledge and implications for genetic counselling. Eur. J. Hum. Genet 17, 722-731 (2009).

46 Antoniou, A. C. \& Easton, D. F. Polygenic inheritance of breast cancer: implications for design of association studies. Genet. Epidemiol. 25, 190-202 (2003).

47 Nichols, J., Zevnik, B., Anastassiadis, K., Niwa, H., Klewe-Nebenius, D., Chambers, I. et al. Formation of pluripotent stem cells in the mammalian embryo depends on the POU transcription factor Oct4. Cell 95, 379-391 (1998).

48 Herr, W., Sturm, R. A., Clerc, R. G., Corcoran, L. M., Baltimore, D., Sharp, P. A. et al. The POU domain: a large conserved region in the mammalian pit-1, oct-1, oct-2, and Caenorhabditis elegans unc-86 gene products. Genes Dev. 2, 1513-1516 (1988).

49 Pesce, M. \& Scholer, H. R. Oct-4: gatekeeper in the beginnings of mammalian development. Stem Cells 19, 271-278 (2001).

50 Wang, P., Branch, D. R., Bali, M., Schultz, G. A., Goss, P. E. \& Jin, T. The POU homeodomain protein OCT3 as a potential transcriptional activator for fibroblast growth factor-4 (FGF-4) in human breast cancer cells. Biochem. J. 375, 199-205 (2003).

51 Conaway, R. C. \& Conaway, J. W. General transcription factors for RNA polymerase II. Prog. Nucleic. Acid Res. Mol. Biol. 56, 327-346 (1997).

52 Hampsey, M. Molecular genetics of the RNA polymerase II general transcriptiona machinery. Microbiol. Mol. Biol. Rev. 62, 465-503 (1998).

53 Amati, B., Dalton, S., Brooks, M. W., Littlewood, T. D., Evan, G. I. \& Land, H. Transcriptional activation by the human c-Myc oncoprotein in yeast requires interaction with Max. Nature 359, 423-426 (1992).

54 Kretzner, L., Blackwood, E. M. \& Eisenman, R. N. Myc and Max proteins possess distinct transcriptional activities. Nature 359, 426-429 (1992).

$55 \mathrm{Gu}$, W., Cechova, K., Tassi, V. \& Dalla-Favera, R. Opposite regulation of gene transcription and cell proliferation by c-Myc and Max. Proc. Natl Acad. Sci. USA 90, 2935-2939 (1993).

56 Lüscher, B. \& Larsson, L. G. The basic region/helix-loop-helix/leucine zipper domain of Myc proto-oncoproteins: function and regulation. Oncogene 18, 29552966 (1999).

57 Mao, D. Y., Watson, J. D., Yan, P. S., Barsyte-Lovejoy, D., Khosravi, F., Wong, W. W. et al. Analysis of Myc bound loci identified by $\mathrm{CpG}$ island arrays shows that Max is essential for Myc-dependent repression. Curr. Biol. 13, 882-886 (2003).

58 Steiger, D., Furrer, M., Schwinkendorf, D. \& Gallant, P. Max-independent functions of Myc in Drosophila melanogaster. Nat. Genet. 40, 1084-1091 (2008).

Supplementary Information accompanies the paper on Journal of Human Genetics website (http://www.nature.com/jhg) 